\title{
A three-phase simulation of the effect of microstructural features on semi-solid tensile deformation
}

\author{
A.B. Phillion ${ }^{1}$, S.L. Cockcroft ${ }^{1}$, and P.D. Lee $^{2}$ \\ ${ }^{1}$ Department of Materials Engineering, University of British Columbia, Vancouver CA \\ ${ }^{2}$ Department of Materials, Imperial College London, London, UK
}

Keywords: microstructure; finite element simulation; semisolid; deformation structure

\begin{abstract}
A direct finite element microstructure model for prediction of the deformation behavior of semisolid metallic alloys is presented. The 2D model geometry is based on a modified Voronoi tessellation, and includes rounded corners to approximate an equiaxed-globular grain structure, liquid surrounding the grains, and micro porosity. An elasto-plastic empirical constitutive equation is derived for the solid grains, while the liquid is approximated as a perfectly plastic material with a very low yield stress. The resulting three-phase model was used to investigate the effects of fraction solid, porosity, and grain size on the constitutive behavior of a semi-solid aluminum alloy, AA5182. The model predictions were validated against experimental data at high fraction solid. These simulations reveal a strong correlation between semi-solid grain size and yield stress, and between porosity and strain localization. The application of direct finite element simulations is shown to be an effective technique for examining the effects of microstructure phenomena on the macro constitutive behavior of semi-solid materials.
\end{abstract}

\section{Introduction}

Over the past few years, a number of new high-strength aluminum alloys have been developed for the automotive and aerospace sectors. Although these alloys are increasingly being used to replace traditional steel components, their wide-range usage remains low because of high production costs. This limited economic viability is due in part to quality issues arising during the solidification processing stage, resulting in decreased productivity and reduced yields. These quality issues occur because of the shrinkage associated with the solid to liquid phase change, the presence of intermetallics, solute segregation, etc. and lead to defects such as macro / micro porosity, and hot tearing. In order to improve the quality of industrially cast components, new experimental and theoretical knowledge is needed. This new knowledge is difficult to obtain due 
to the high temperatures associated with castings, the presence of a liquid phase, and uncertainty in the relationship between fraction solid and temperature.

One methodology which has not previously been explored in semi-solids is the combination of experimentally derived measurements and a direct finite element (FE) microstructure simulation. The combination of these two techniques promises to be a powerful tool, producing a validated model to perform virtual experiments and significantly extend the range of conditions that may be examined. In other research areas, a number of modeling studies have been reported which link microstructural features to the macro constitutive behavior [1-8]. These studies include the prediction of 3D polycrystalline microstructure with crystallographic orientation and secondary phase particles by combining 2D micrographs with a modified 3D Voronoi tessellation function [6-8], along with the prediction of fatigue behavior of multi phase material by coupling simulations of micro-plasticity in idealized microstructure with experimental measurements [5]. However, such studies on semi-solid metals do not yet exist.

The extension of the direct FE simulation technique to a study of a three-phase semi-solid has been made possible by combining the above techniques with new studies which have modeled the evolution of microstructure and fluid flow during solidification. The key difficulty has been in characterizing the semi-solid microstructure. However, in work by Mathier et al. [9], and subsequently by Vernède et al. [10,11], this difficulty was addressed through the application of a granular model using the Voronoi tessellation technique to model the solidification of globular grains. In this technique, grains are approximated as polyhedrons based on a Voronoi tessellation of the nuclei centers. This tessellation describes the area of points closest to each nuclei, with borders halfway between neighboring nuclei. Solidification is carried out by advancing the grain edges towards the border along a linear segment connecting the nuclei with a Voronoi vertex. The results of $[10,11]$ showed that solidification occurs via the progressive formation of grain clusters. Furthermore, the semi-solid morphological transitions, and the localization of liquid feeding at high fraction solid appears to be strongly influenced by cooling rate.

While granular models have previously been used to describe semi-solid ductility $[12,13]$ and liquid feeding [14], these previous works assumed a regular arrangement of grains. In contrast, the use of a Voronoi tessellation allows for examination of a random grain geometry and thus better simulates real semi-solid microstructure. For example, the microstructure derived from a 
granular model for an $\mathrm{Al}-1 \mathrm{wt} \% \mathrm{Cu}$ alloy has been shown to closely match the predictions made using the phase-field method but with substantially less computational cost [11].

The granular solidification model of Vernède et al. [10,11] was previously used to gain insight into one of the major industrial solidification defects: hot tearing. Although hot tearing is known to be affected by a number of microstructural and processing factors, efforts to quantitatively predict this defect have met with only limited success [15]. There are several requirements that must be met in order to improve this predictive capability. Firstly, one must have a quantitative understanding of semi-solid tensile constitutive behavior. Secondly, one must have a means of quantifying the development of semi-solid stress or strain subject to a prevailing set of process conditions. Thirdly, one must be able to link the strain to the accumulation of damage prior to failure. While a number of recent experimental studies have been designed to investigate these requirements [15-24], a comprehensive understanding linking microstructural features to semi-solid constitutive behavior is needed to improve hot tearing criteria.

In the current work, a 2D model is described that examines the effect of microstructure on the elasto-plastic behavior of semi-solid materials at high fraction solid $\left(0.75<f_{\mathrm{s}}<0.95\right)$. The semisolid microstructure is characterized via geometry based on a Voronoi tessellation, and includes the following features: randomly distributed primary grains; liquid at the boundary between grains with a volume fraction similar to $f_{\text {liq }}$; variable void size; and variable fraction porosity. Using this characterization of the semi-solid microstructure, direct FE simulations are performed to numerically predict the semi-solid constitutive behavior across a wide range of microstructural conditions. Finally, this novel three-phase direct simulation technique is validated against experimental semi-solid tensile constitutive behavior using data for two aluminum alloys, AA5182 and AA6111.

\section{Model Development}

Firstly, a methodology for generation of the three-phase semi-solid microstructure is presented using a granular approach similar to the solidification model proposed by Mathier et al. [9]. Secondly, the constitutive behavior of the solid and liquid phases, as well as the mesh, boundary conditions, and assumptions in the FE simulations are described. 


\section{a. Generation of Microstructural Geometry}

While the Voronoi tessellation approach used by Mathier et al. [9] was used to model solidification from $f_{\mathrm{s}}=0$ to $f_{\mathrm{s}}=1$, the present work creates semi-solid microstructure at a specific fraction solid. In order to match the model geometry to semi-solid microstructure obtained during casting processes, two additional features have been included: (1) rounded grain corners, and (2) as-cast porosity. Rounded corners are needed because the geometry generated directly from the Voronoi tessellation leads to polyhedral grains with sharp corners. Sharp corners do not exist during solidification due to surface tension effects, and will produce unrealistic stress concentrations. As-cast porosity is needed because it is an important microstructural phenomena, and was shown previously to be highly relevant to the problem of hot tearing [18, 25, 26].

Creation of the equiaxed-globular geometry consists of the following steps: producing a 2D Voronoi diagram; reducing the grains in size based on the fraction solid; rounding the grain corners via the Gibbs-Thomson effect; and adding porosity at the triple-junctions.

\section{Creation of the Voronoi Diagram}

The Voronoi tessellation is assembled using the qhull [27] program. This program requires as input the number of discrete points, or grain nuclei, and the bounding box which encloses these grain nuclei. The number of discrete points is calculated based on the required equivalent grain size assuming circular grains. The output is a single text file containing the region numbers and the coordinates of the vertices making up the perimeter of each region. A Voronoi diagram for nine nuclei randomly placed in a $0.15 \mathrm{~mm}^{2}$ domain is shown in Fig. 1(a).

\section{Accounting for the Fraction Solid}

The geometry shown in Fig. 1(a) represents a fully solidified microstructure. To model the semisolid at a specific fraction solid, the grains are reduced in size and the region between grains is assumed to be liquid. The predicted geometry for a fraction solid of $f_{\mathrm{s}}=0.90$ is shown in Fig. 1(b), with the liquid region is represented by the dark shade. Each grain is reduced in size independently of the others by moving the coordinates of each vertex towards the nucleation point until the area of this new grain relative to the area of the Voronoi region is equal to the fraction solid of interest. The resulting geometry consists of a random configuration of grains fully surrounded by a continuous film of liquid. The liquid channels between the grains vary in thickness depending on the size of the grains, and the distance between the two grain nuclei in 
any particular channel. The relationship between fraction solid and temperature used in this work to model semi-solid AA5182 was taken from Thompson et al. [28], and is provided in Table A.

\section{$\underline{\text { Rounding the Grain Corners }}$}

The geometry shown in Fig. 1(b) does not yet represent a semi-solid microstructure because the solid grains are unrealistically shaped as sharp-cornered polygons. To improve the semi-solid microstructure geometry, the grain corners are rounded using a solute flux model previously developed by Vernède et al. [11]. This geometry is shown in Fig. 1(c).

The solute flux model is based on a balance between solute flux induced by the GibbsThomson effect and the geometrical advantage of a rounded corner for diffusion. Since the globular grains are not experimentally found to be spherical during solidification, the grain corners are modeled by a curved boundary with radius $R$, while elsewhere the boundary is flat and parallel to a Voronoi edge segment. Furthermore, since the rounded interface increases the overall volume of liquid at the triple-junctions, the grain vertices must be moved slightly forward to maintain a constant fraction solid.

\section{Adding Porosity}

The last step in constructing the semi-solid microstructure is to add porosity. There are four variables that must be included: size distribution, shape, area fraction, and location. These variables were previously quantified in [29] and [30] in as-cast aluminum alloys using x-ray micro-tomography. This previous work showed that as-cast porosity is highly tortuous in shape, with a size distribution ranging from $\sim 2$ to $175 \mu \mathrm{m}$ in equivalent radius, a volume fraction ranging from 0.003 to 0.006 , an average radius of $7.5 \mu \mathrm{m}$, and generally located at the triplejunctions.

Porosity is added to the model geometry by removing material from the liquid at both the triple-junctions and the midpoint of a liquid channel between two Voronoi vertices, as shown in Fig. 1(d) (the pores are represented by the grey circles). The size of these voids is $7.5 \mu \mathrm{m}$ in radius. Note that material is only removed from the liquid portion of the geometry and thus voids placed in triple-junctions less than $7.5 \mu \mathrm{m}$ in radius will be irregularly-shaped. For example, the enlarged void in Fig. 1(d) is irregularly-shaped because the triple-junction radius at that location was smaller than $7.5 \mu \mathrm{m}$. In contrast, some of the other voids in Fig. 1 (d) are 
circular because they were placed in larger triple-junctions. In order to accommodate the porosity, the fraction liquid decreases, while the fraction solid stays the same assuming that $f_{\mathrm{s}}+f_{1}+f_{\mathrm{p}}=1$. This assumption may induce a small error in the results since at constant $f_{\mathrm{s}}$ the ratio of $f_{1} / f_{\mathrm{s}}$ is not constant with varying $f_{\mathrm{p}}$.

\section{b. Finite Element Model}

The methodology outlined above provides the model geometry necessary for direct FE simulation of semi-solid tensile deformation. A script file was written in the Python programming language to create this geometry within the ABAQUS ${ }^{1}$, framework based on three user-input variables: fraction solid, fraction porosity, and equivalent grain size, $\bar{d}$. The equivalent grain size is defined as the grain diameter assuming that all grains are the same size and are circular in shape, as per Eq. (1), where $D^{2}$ is the area of the REV. Once the geometry exists, the FE model can be constructed by determining appropriate material properties, applying loads and boundary conditions, and generating a mesh.

$$
\bar{d}=\sqrt{\frac{4 D^{2}}{N_{g} \cdot \pi}}
$$

\section{$\underline{\text { Material Properties }}$}

The input constitutive behavior of the solid and liquid phases is a critical aspect of the semisolid microstructure model. The mechanical properties required for the analysis include Poisson's Ratio, elastic modulus and the stress-strain behavior for both the solid and the liquid. In this model, the constitutive behavior of the solid grains is described as an elasto-plastic material, while the liquid is described as a perfectly plastic material.

Elastic Modulus -The choice of elastic modulus for the liquid constituent is critical because the liquid network is fully interconnected, and is much weaker than the solid. A liquid modulus of $0.7 \mathrm{GPa}$ was chosen to offer little resistance to stress. The modulus of the solid grains was assumed to be $70 \mathrm{GPa}$, that of a low temperature aluminum alloy.

Poisson's Ratio - To conserve volume within the liquid, Poisson's ratio, v, should be equal to 0.5. However, this value cannot be used in the FE analysis because the resulting stiffness matrix

\footnotetext{
${ }^{1}$ SIMULIA, Providence, RI, USA
} 
will contain a zero in the denominator. By trial and error, $v=0.45$, provides a compromise between conserving volume and minimizing convergence issues. In the solid grains, $v=0.30$.

Flow Stress Behavior of the Solid - To provide the required solid flow stress behavior, an empirical constitutive equation for an as-cast aluminum-magnesium alloy, AA5182, was used. The equation is based on the work by Chaudhary [31] but has been modified for use at high temperatures using flow stress measurements taken from [18] of hot isostatic pressing (HIP) AA5182 material in the temperature range 500 to $560^{\circ} \mathrm{C}$. Furthermore, a lower bound of 1.5 MPa was applied to ensure sufficient differentiation between the behavior of the solid and the liquid. The equation was modified using experimental data from HIP material because it remains fully solid at temperatures above the normal as-cast solidus temperature, due to chemical homogenization. This allowed for direct measurement of the flow stress of the solid grains at temperatures where the material is normally semi-solid. However, note that the composition of the HIP material will vary slightly as compared to the as-cast material since it uniformly contains the bulk composition whereas as-cast material will have a compositional gradient due to the dendritic solidification [18].

The empirical constitutive equation is based on an extended Ludwik formulation:

$$
\sigma(\varepsilon, \dot{\varepsilon}, T)=K(\varepsilon, \dot{\varepsilon}, T) \cdot \varepsilon^{\mathrm{n}(\mathrm{T})} \cdot \dot{\varepsilon}^{\mathrm{m}(\mathrm{T})}
$$

where $\sigma$ is the stress, $\varepsilon$ is the total plastic strain experienced by the material, $\dot{\varepsilon}$ is the strain rate, $K$ is a flow stress coefficient, $n$ is the strain hardening parameter and $m$ is strain rate sensitivity of the material. These parameters all vary as a function of temperature, and thus the equation is able to predict the constitutive behavior from room temperature up to the mechanical coherency temperature over a large range of strain and strain-rate. The correlations developed by Chaudhary for $m, n$, and $K$ are shown in Table B. Also shown in Table B are the correlations for the modified empirical equation. The modification consisted of adjusting the $y$-intercept of the $K$ parameter correlation by $\sim 5 \%$. The $m$ and $n$ parameter were not changed. Note that above $350^{\circ} \mathrm{C}, n$ was found to have a value of zero, i.e. a material which is elastic perfectly plastic.

A comparison of the flow stresses predicted by Chaudhary's empirical equation, the modified empirical equation, and the experimental measurements from [18] is provided in Fig. 2. As can be seen from Fig. 2, the Ludwik equation yields a much better correlation to the experimental 
data using the modified $K$ as compared to the base-line equation. This result is not surprising, since Chaudhary's empirical equation was developed for the temperature range $25-470^{\circ} \mathrm{C}$.

Flow Stress Behavior of the Liquid - For small liquid channels, such as semi-solid material at high fraction solid, the liquid flow stress will depend on both the channel thickness and the solidliquid interfacial energy [12], and will very between two extremes. The lower bound of this deformation behavior is the viscous flow of a lubricating fluid, although this can lead to significant shear stresses. The upper bound is the stress necessary to separate in tension two plates bonded by a thin liquid film. This stress may be calculated from the Young and Laplace equation [12]:

$$
\sigma_{\ell}=2 \gamma_{\mathrm{sl}} / \mathrm{h}
$$

where $\sigma$ is the capillary stress, $\gamma_{\mathrm{sl}}$ is the solid-liquid interfacial energy $\left(\sim 1 \mathrm{~J} \mathrm{~m}^{-2}\right.$ for $\mathrm{Al}$ alloys), and $h$ is the thickness of the liquid film. Based on the range of fraction solids, grain sizes, and the corresponding liquid channel thicknesses used in this work [13], the calculated capillary stress based on Eq. (3) ranges from 0.2 to $1.6 \mathrm{MPa}$. However, in this case, the channel thickness $h$ is not uniform for a specific grain size and fraction solid, but varies due to the randomness of the grain structure imposed by the Voronoi tessellation. Based on these challenges, the liquid behavior was assumed to be perfectly plastic with a flow stress of $0.5 \mathrm{MPa}$. The implications and limitations associated with the use of a perfectly plastic constitutive model for the liquid will be discussed in Section IV.

\section{Constraints and Loading}

The model domain is a 2D square geometry. Symmetry boundary conditions are imposed on the left and bottom sides of the model domain, while the right side is free to move in all directions. The top side of the model domain is linked to a reference node. Thus, the model is constrained as though it were a quarter-section of a plate of material. To deform the model, a displacement is applied to the reference node corresponding to a bulk strain of 0.0225 at a strain rate of $0.0015 \mathrm{~s}^{-1}$. This allowed the bulk semi-solid constitutive behavior to be obtained from the predicted force-displacement curve at the reference node. Note that the liquid nodes are not constrained by the boundary conditions, to facilitate liquid flow both in and out of the liquid channels bordering the domain boundary. 


\section{$\underline{\text { Finite Element Mesh }}$}

The FE mesh was constructed from the above geometry. The solid grains were meshed with a combination of triangular and quadrilateral plane strain elements, while the liquid films were meshed with triangular plane strain elements. The average liquid element length was $2 \mu \mathrm{m}$. From the results of a sensitivity analysis, to be presented in Section $I V$, a value of $5 \mu \mathrm{m}$ was chosen as the average solid element edge length.

The size of the representative elementary volume (REV), which dictates the number of grains that are included in the simulation $\left(N_{\mathrm{g}}\right)$, also had a large effect on the predicted constitutive behavior. This is due to the random nature of the Voronoi tessellation, which for a specified $N_{\mathrm{g}}$ will alter the geometric configuration of the grains based on the randomly generated grain nuclei. At low values of $N_{\mathrm{g}}$, the number of liquid channels is too low, and the results will be biased to that of the solid rather than of a semi-solid mixture. Based on a sensitivity analysis, presented in Section $I V$, the size of the model domain was 56 grains.

\section{Results and Discussion}

The three-phase direct FE simulation was run under a variety of scenarios in order to predict the tensile deformation behavior of a semi-solid aluminum alloy. Firstly, results showing the effect of fraction solid on constitutive behavior are presented. Secondly, a sensitivity analysis of some of the critical model assumptions and the model limitations are discussed. Thirdly, the model is validated via a comparison to experimental-determined semi-solid constitutive behavior and also to ductility measurements [31]. Finally, unique results are presented whereby the model is used to explore the effects of porosity and grain size on semi-solid constitutive behavior. The stress-strain predictions are based on the force-displacement curves provided by the finite element software for the reference node. The reference node was displaced an amount equivalent to a strain $\varepsilon=0.0225$. However, in many of the simulations, convergence issues arose which prevented the model from reaching this level of deformation.

\section{a. Model Predictions}

The effect of fraction solid on the stress-strain predictions in the range $0.75<f_{\mathrm{s}}<0.95$ is

shown in Fig. 3 in the absence of porosity and for an equivalent grain size, $\bar{d}$, of $225 \mu \mathrm{m}$. Fraction solids less than $f_{\mathrm{s}}=0.75$ were not examined because the assumption that the liquid 
phase behaves as a semi-continuous film is no longer valid. As can be seen in the figure, the semi-solid flow stress decreases with decreasing fraction solid. This stress decrease is related to changes occurring in both the solid grains and the liquid film. In the solid grains, there is a decrease in flow stress due the effect of increasing temperature. In the liquid, decreasing the fraction solid corresponds to thicker liquid channels which allow for more strain accumulation in the liquid before the solid grains begin to interlock.

The predictions made by the semi-solid microstructure model and shown in Fig. 3 can be described as elastic-plastic with no well-defined yield point, and considerable hardening. This behavior is not the classical work hardening effect, but rather geometric hardening due to an increasing number of grains interacting with each other instead of freely moving within the liquid. Non-classical work hardening has previously been experimentally observed in high fraction solid semi-solid aluminum alloys (AA5182 [32] and Al-2wt\% Cu [17]) under tensile loading and after re-heating the specimen from room temperature. However, in these earlier works it was not possible to transform the force-displacement measurements into stress-strain behavior since the length over which deformation takes place was not known.

The microstructure model is able to simulate the constitutive behavior in semi-solid as-cast material under conditions that would normally be a challenge to measure experimentally. The data shown in Fig. 3 provides new knowledge regarding semi-solid deformation, such as a mechanism for the experimentally-observed semi-solid work hardening effect [17, 32], flow stress at $f_{\mathrm{s}}<0.95$, and the effect of the solid on semi-solid behavior. For example, although there is much liquid present at $f_{\mathrm{s}}=0.85$, the bulk stress-strain curve shown in Fig. 3 exhibits a flow stress significantly higher than that of the liquid alone.

\section{b. Sensitivity Analysis and Model Limitations}

During the development of the semi-solid microstructure model, a number of critical quantitative assumptions were required in formulating the model. The effects of these assumptions on the model predictions, as well as model limitations, are discussed below.

Firstly, the effect of liquid flow stress on the predicted semi-solid stress-strain curve was investigated by performing four simulations, with varying liquid flow stress between 0.1 and 1.5 MPa. For this analysis, $f_{\mathrm{s}}=0.95$ and $T=570^{\circ} \mathrm{C}$. The results indicate that the bulk properties are highly influenced by the choice of liquid flow stress. For example, the bulk flow stress at 
$\varepsilon=0.004$ is predicted to increase from 5.83 to $6.32 \mathrm{MPa}$ in conjunction with an increase in the liquid flow stress from 0.5 to $1.5 \mathrm{MPa}$. At flow stress values lower than $0.5 \mathrm{MPa}$ (e.g. 0.1 MPa), numerous convergence issues arise.

Secondly, the effect of element size on the predicted semi-solid stress-strain curve was investigated by performing six simulations, with a varying average solid element edge length between $2 \mu \mathrm{m}$ and $32 \mu \mathrm{m}$. For this analysis, $f_{\mathrm{s}}=0.90$ and $T=580^{\circ} \mathrm{C}$. Table $\mathrm{D}$ shows both the variation in predicted stress at $\varepsilon=0.01$, and the standard deviation in the range $0<\varepsilon<0.01$. The standard deviation was calculated by comparing the stress-strain curve for the simulation with a solid element edge length of $2 \mu \mathrm{m}$ with the predictions obtained using larger elements. The results indicate that the solid element size has a large effect on the predicted bulk flow stress, with larger elements predicting higher semi-solid stresses. The standard deviation is also seen to decrease monotonically with decreasing element sizes, and is less than $2 \%$ when the element size is $5 \mu \mathrm{m}$ or less. Hence, the solution appears to be converging on an exact value.

Thirdly, the effect of representative elementary volume size on the predicted constitutive behavior was investigated through analysis of the relationship between geometric configuration and model predictions. In Fig. 4, stress-strain predictions are presented from 25 different simulations of 56 grains $-f_{\mathrm{s}}=0.90$ and $\bar{d}=150 \mu \mathrm{m}$ - each with a different geometric configuration. As can be seen in the figure, the different geometric configurations provide stochastic variability to the constitutive behavior. For example, at $\varepsilon=0.01$, the flow stresses vary between $\sim 4$ and $5 \mathrm{MPa}$ (corresponding to a maximum flow stress variation, $\Delta \sigma$, of $0.98 \mathrm{MPa}$ ). This analysis was repeated for $N_{\mathrm{g}}=14,28,56$, and 100. The variation in $\Delta \sigma$ is shown in Table C, and is significantly larger with 14 or 28 grains in the model domain as compared to 56 grains, and is slightly less for 100 grains.

The choice of element size of $5 \mu \mathrm{m}$ and $N_{\mathrm{g}}=56$ grains were made as a compromise between simulation run time and accuracy. Based on the sensitivity analysis, these choices appear reasonable considering many of the inherent assumptions within the model. For example, in the case of simulated number of grains, increasing the model domain to 100 grains would decrease the variation in stress-strain response due to geometric variability by approximately fifteen percent. However, this improvement would be offset by a twofold increase in the number of elements required to model the larger domain, and a four-fold increase in computation time from 
$\sim 4$ to $16+$ hours. The choice of liquid flow stress, $\sigma=0.5$, seems somewhat more arbitrary and certainly has a significant effect on the stress-strain predictions. This value was chosen based on calculations from Eq. (3), and the need to have a value that is much lower than the solid flow stress while avoiding convergence issues.

There are also a number of model limitations. One major limitation relates to the use of a perfectly plastic constitutive model for the liquid phase. The implications of this assumption are threefold. Firstly, the liquid displacement occurs via element deformation rather than fluid flow. This means that the extent to which bulk flow can occur from channels undergoing contraction to channels undergoing expansion is severely limited. Secondly, the model predictions are valid only at low values of strain, since to achieve the large strains in the liquid seen in Fig. 6(b) the elements must be significantly deformed and are approaching or exceeding their distortion limit. Thirdly, the use of a yield stress linked to a tensile force instead of a shear stress linked to the viscosity and fluid velocity results in all the liquid channels behaving identically. In reality, each of these channels will behave differently due to the varying channel thickness and the bulk permeability of the REV. A second limitation is the 2D geometry. In reality, solidification microstructures have a complex 3D geometry. The 2D model geometry will predict grain interlocking earlier than a 3D structure and thus the current 2D model predictions will be stiffer. This is because only one phase (either the solid or the liquid) can be continuous. In contrast, 3D structures allow for simultaneous continuity in both phases. A third limitation relates to void coalescence. In the real situation, new voids will form and the existing voids will grow and coalesce. This effect has not been incorporated into the current finite element model and yet is known to have a large impact on strain localization and material failure by hot tearing [23].

\section{c. Model Validation}

In order to validate the semi-solid microstructure model, the model predictions in the absence of porosity have been compared to three experimentally-obtained semi-solid stress-strain curves of HIP AA5182. The experimental methodology has been detailed elsewhere [18]. This data was obtained at a set-point temperature of $570^{\circ} \mathrm{C}$, yet the data showed a rather large variability in the flow stress $\left(2.7,4.1\right.$, and 9.5 MPa), and failure strain $\left(1.7 \times 10^{-4}, 1.4 \times 10^{-4}\right.$, and $\left.3.2 \times 10^{-3}\right)$. Due to the experimental methodology, it is not unreasonable to assume that the actual test temperature may have been a few degrees higher or lower than the set-point value of $570^{\circ} \mathrm{C}$. This small 
temperature variation will greatly alter the semi-solid structure, including reducing the number of grain bridges and increasing the number of grains completely wetted by liquid films. In turn, these changes in microstructure will significantly modify the measured constitutive behavior.

To examine this data, three different model simulations were conducted, corresponding to $f_{\mathrm{s}}=0.95,0.98$, and 1.0. For this analysis, $f_{\mathrm{p}}=0$, and $\bar{d}=225 \mu \mathrm{m}$. A comparison of the experimental measurements and the model predictions is shown in Fig. 5. An enlargement of this figure for the range $\varepsilon<0.00035$ is also provided for $f_{\mathrm{s}}=0.95$ and 0.98 . Note that the experimental data for $f_{\mathrm{s}}=0.95$ and $f_{\mathrm{s}}=0.98$ is available only over a limited range since the material contained little ductility. As can be seen in the figure, the model results reproduce the bulk behavior when the system is fully solid $\left(f_{\mathrm{s}}=1\right)$, and at relatively higher values of strain $(\varepsilon>$ 0.001). The sharp yield point predicted by the model occurs because the constitutive behavior is given as elastic perfectly plastic. However, the experimental data appears to exhibit some strain hardening and no well-defined yield point. This is thought to have occurred because the fraction solid in the experiment was very high, and thus the specimen would have consisted of small and isolated pockets of liquid. Unfortunately, the semi-solid microstructure model does not support the range $0.98<f_{\mathrm{s}}<1$ due to a highly distorted mesh which does not convergence. As can be seen in both the main graph and in the enlarged figure, the stress-strain predictions for $f_{\mathrm{s}}=0.95$ and $f_{\mathrm{s}}=0.98$ also match quite well the experimental measurements, up to the point of fracture. Assuming a semi-solid bulk elastic modulus of $35 \mathrm{MPa}$ (i.e. half the value of a low-temperature aluminum alloy), fracture occurred within the plastic portion of the curve between $2 x$ and $3 x$ the strain required for yielding. It is not possible to provide a bulk yield strain for the model since the liquid and each of the solid grains will yield at different values of bulk strain. The model predictions are thus able to capture the changes in experimental behavior in both the elastic and plastic regimes simply by varying the fraction solid. Therefore despite relatively large assumptions in its formulation, the semi-solid microstructure model seems to satisfactorily predict the constitutive behavior of AA5182.

The model also allows for the examination of the local distribution of strain and the limits in ductility, shown in Fig. 6, which can be compared to the experimental data of a prior author. Using an optical strain technique, Mitchell et al. [24] measured surface strain during solidification in a constrained casting for the aluminum-magnesium-silicon alloy AA6111, along a line bisecting a hot tear. These prior results are provided in Fig. 6(a), and indicate that 
the bulk material is able to accommodate strains in the range of 0.002 to 0.004 before strain localization, and that hot tears began to form when the local strain reaches a value of 0.016 to 0.019. As can be seen in the figure, the strain development is initially randomly distributed. With increasing time, the strain becomes localized in a few areas until at $4.4 \mathrm{~s}$ all the strain is accommodated in the region where a hot tear forms.

A similar technique can be used to investigate the strain distribution in the semi-solid microstructure model. Fig. 6(b) shows the local strain as a function of distance along a line perpendicular to the loading direction (at mid-height along the geometry), for various times after deformation start similar to Fig. 6(a). The simulation was performed with $f_{\mathrm{s}}=0.90, f_{\mathrm{p}}=0$, and $\bar{d}=150 \mu \mathrm{m}$. As can be seen in the figure, there are four regions of strain concentration, corresponding to the liquid channels and a number of regions of low strain, corresponding to solid grains. The two highest peaks represent triple-junction locations.

In comparing the experimental data and the simulation results, there are a few salient observations that may be made. Firstly, the results presented in Figs. 6(a) and 6(b) are qualitatively similar, with both figures exhibiting regions of strain localization that evolve with time. Secondly, the magnitude of strain predicted by the simulation is an order of magnitude larger than the strain accumulated in the experimental data prior to failure. Thirdly, the simulation has small regions of localized strain, $\sim 25-100 \mu \mathrm{m}$, while experimental data shows that strain is localized on a length scale of $\sim 400-800 \mu \mathrm{m}$. Finally, the local strain in some of the simulated triple-junctions $(\varepsilon \sim 0.3-0.4)$, far exceeds the local strain measurements $(\varepsilon \sim 0.016-$ 0.019) made by Mitchell et al. [24].

Much of the difference between the experimental data and the semi-solid microstructure model with respect to strain distribution may be attributed to the fact that the model is predicting results for an isothermal or iso-fraction solid test in which the level of damage does not evolve with time, whereas the experimental results show the evolution in strain for non-isothermal material undergoing solidification and accumulation of internal damage. In reference to the localized region size, the model is able to support strain localization in most of the liquid channels. In contrast, it was hypothesized in [29] and [30] that localization of internal damage during semisolid tensile deformation occurs within only a few of the liquid channels. It is also possible that the experimental results are not resolving strain localization within individual liquid channels, but rather within grain clusters, i.e. the groupings of grains proposed by Vernède that are fully 
surrounded by liquid films [10]. These grain clusters may have the capacity to both localize strain to within the cluster, and to partition strain between solid and liquid. In reference to the strain accumulation prior to failure, it is clear from the experimental data in [18] and [24] that the model need only to simulate the semi-solid deformation conditions at small strains. Therefore, it provides a good qualitative description of the local strain accumulation in semi-solid materials.

\section{d. The Effect of Grain Size and Porosity on Model Predictions}

In Fig. 7, the results of a 'virtual' experiment, showing the effects of grain size and porosity on semi-solid constitutive behavior, are presented. These results are unique since these effects cannot easily be quantified using experimental techniques. For the grain size assessment, Fig. 7 (a), the simulation was run with the following parameters: $T=580^{\circ} \mathrm{C}, f_{\mathrm{s}}=0.90, \bar{d}=75,150$, 225 , and $300 \mu \mathrm{m}$, and $f_{\mathrm{p}}=0$. As shown in the figure, the semi-solid flow stress decreases with increasing grain size. This decrease occurs because for a given fraction solid, larger gains must necessarily have thicker liquid films surrounding the grain boundary. These thicker films allow for more deformation in the liquid before the grains interact with each other. Grain size effects have also been seen in previous micro-models (e.g. [33-35]), such as Venkatramani et al., [33] in which effect of microstructure size on the mechanical response of two-phase polycrystalline Ti6242 alloy is studied, and the Hall-Petch relation is reproduced. This is the first work, however, to demonstrate the importance of grain size on semi-solid deformation mechanisms.

For the fraction porosity assessment, presented in Fig. 7(b), the simulation was run with the following parameters: $T=560^{\circ} \mathrm{C}, f_{\mathrm{s}}=0.95, \bar{d}=75,150 \mu \mathrm{m}$, and $f_{\mathrm{p}}=0,0.002,0.004$, and 0.006 . The results indicate that as-cast porosity will decrease the flow stress of the semi-solid material, though the effect is relatively small in comparison to the effect of grain size. This is because porosity at the triple-junctions creates internal surface area within the model, which allows the liquid to flow more easily in response to the applied deformation. The porosity has the secondary effect of strain localization. This effect is evident in Fig. 8, which shows strain contours from the model run with $f_{\mathrm{p}}=0.004$. As can be seen in the figure, strain localization has occurred in the liquid area between voids $A$ and $B$. It is clear that this localization would cause the two voids to preferentially grow in the interligament region and coalesce to form a single larger void. While strain localization and associated void coalescence is a common feature within ductile materials [36], its effect here is significantly increased because of the weak liquid channels between the 
grains. The consequence of ignoring the process of coalescence significantly affects the effect of porosity on semi-solid constitutive behavior. However, this feature cannot be added into the model geometry as currently formulated. Fig. 8 also highlights two other semi-solid deformation phenomena captured by the model: shear banding on some of the grains at higher strains, and the accumulation of strain along the liquid channels at approximately $45^{\circ}$ to the loading direction. Thus, similarly to what was seen by Gao et al. in terms of fatigue resistance [5], it would appear that the presence of porosity increases the local stress, and localizes the plastic strain. Furthermore, the location and initial size of the voids will greatly affect the occurrence of hot tearing.

\section{Conclusions}

A novel, three-phase, direct finite element microstructure model is presented which describes the tensile deformation behavior of semi-solid metallic alloys. The model geometry was generated using Voronoi tessellation with rounded corners, producing a two phase equiaxedglobular grain structure. Voids were added at the triple-junctions, representative of as-cast porosity, to produce a three phase simulation. The model was validated against experimentally measured semi-solid stress-strain curves of HIP AA5182, and strain localization measurements during casting of AA6111. Sensitivity analysis was performed to predict the two phase behavior as a function of liquid flow stress, element size, and representative elementary volume size.

The validated model was then applied to predict the effect of fraction solid, fraction porosity, and grain size on three phase semi-solid constitutive behavior. The model correctly predicts the reduction in flow stress associated with increasing grain size or fraction porosity; the mechanism for this reduction is demonstrated to be strain localization within the liquid. Furthermore, the semi-solid deformation behavior is predicted to be most dependent on fraction solid, followed by grain size and then porosity, for the ranges simulated.

The use of direct finite element microstructure simulation is shown to be a valuable tool for investigating the influence of semi-solid constitutive behavior on the formation of defects during industrial metals processing. These three-phase simulations provide, for the first time, direct evidence that strain localization in the liquid results in significantly reduced macroscopic properties, which leads to premature failure. This strain localization in the liquid is strongly influenced by grain size and the presence of porosity. 


\section{Acknowledgements}

The authors would like to acknowledge NSERC, EPSRC (GR/T26344), and Alcan Intl. for financial support, and thank Prof. D. Maijer for fruitful discussions regarding modeling of the liquid phase within the ABAQUS framework.

\section{Tables}

Table A: The relationship between fraction solid and temperature for AA5182 after Thompson et al. [28].

\begin{tabular}{|c|c|}
\hline Temp. $\left({ }^{\circ} \mathbf{C}\right)$ & $\boldsymbol{f}_{\mathbf{s}}$ \\
\hline 561 & 0.95 \\
\hline 578 & 0.90 \\
\hline 588 & 0.85 \\
\hline 596 & 0.80 \\
\hline 602 & 0.75 \\
\hline
\end{tabular}

Table B: AA5182 Ludwik parameter correlations for $K, n$, and $m$ [31].

\begin{tabular}{|c|c|c|}
\hline K (MPa) & $\mathbf{N}$ & $\mathbf{M}$ \\
\hline$T\left({ }^{\circ} C\right)<200$ & $T\left({ }^{\circ} C\right)<150$ & $T\left({ }^{\circ} C\right)<150$ \\
$K=448-0.63 T$ & $n=0.25-0.0005 T$ & $m=0$ \\
$200<T<500$ & $150<T<350$ & $150<T<400$ \\
$K=458.2-0.77 T\}$ Original & $n=0.29-0.0008 T$ & $m=0.1497-0.001 T$ \\
$K=483.5-0.77 T\}$ Enhanced & $350<T<500$ & $400<T<500$ \\
& $n=0$ & $m=0.205+0.00006 T$ \\
\hline
\end{tabular}

Table C: The variation in $\Delta \sigma$ as a function of $N_{\mathrm{g}}$, calculated from 25 different geometric configurations $\left(f_{\mathrm{s}}=\mathbf{0 . 9 0}, \bar{d}=150 \mu \mathrm{m}\right)$.

\begin{tabular}{|c|c|}
\hline $\boldsymbol{N}_{\mathbf{g}}$ & $\boldsymbol{\Delta} \boldsymbol{\sigma} \boldsymbol{\boldsymbol { a }} \boldsymbol{\varepsilon}=\mathbf{0 . 0 1}$ \\
\hline 14 & 2 \\
\hline 28 & 1.5 \\
\hline 56 & 0.98 \\
\hline 100 & 0.82 \\
\hline
\end{tabular}


Table D: The variation in both the predicted stress at $\varepsilon=0.01$ and the standard deviation in stress-strain response as a function of element $\operatorname{size}\left(f_{\mathrm{s}}=0.90, T=580^{\circ} \mathrm{C}\right)$.

\begin{tabular}{|c|c|c|}
\hline $\begin{array}{c}\text { Element } \\
\text { Size }(\mu \mathrm{m})\end{array}$ & $\begin{array}{c}\text { Predicted Stress } \\
\text { at } \varepsilon=0.01(\mathrm{MPa})\end{array}$ & $\begin{array}{c}\text { Standard } \\
\text { Deviation }\end{array}$ \\
\hline 32 & 5.09 & 3.59 \\
\hline 16 & 4.94 & 3.25 \\
\hline 8 & 4.77 & 2.53 \\
\hline 5 & 4.67 & 1.97 \\
\hline 4 & 4.61 & 1.52 \\
\hline 2 & 4.55 & - \\
\hline
\end{tabular}

\section{Figure Headings}

Fig. 1: Schematic showing the stages of the semi-solid microstructure model: (a) Voronoi diagram, (b) $f_{\mathrm{s}}=\mathbf{0 . 9 0}$ with sharp corners, (c) round corners and (d) porosity.

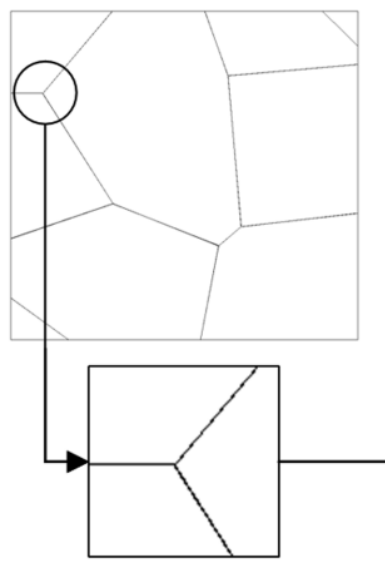

(a)

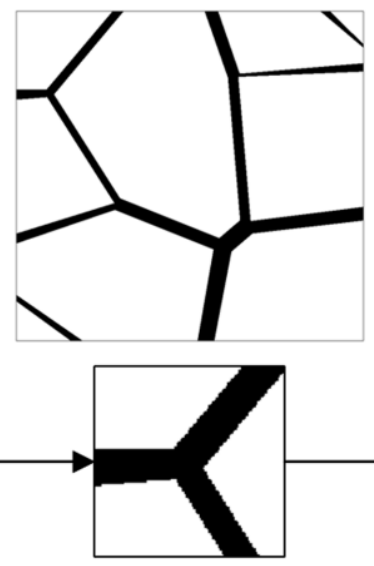

(b)

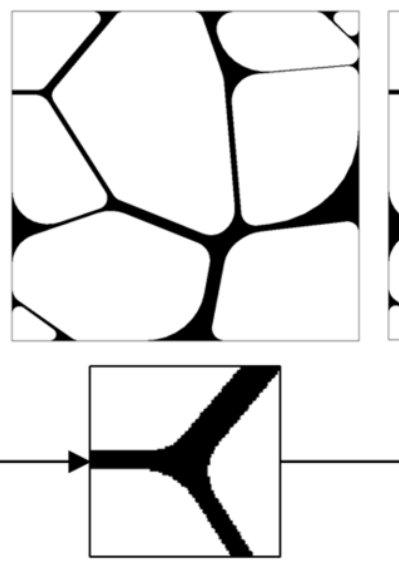

(c)

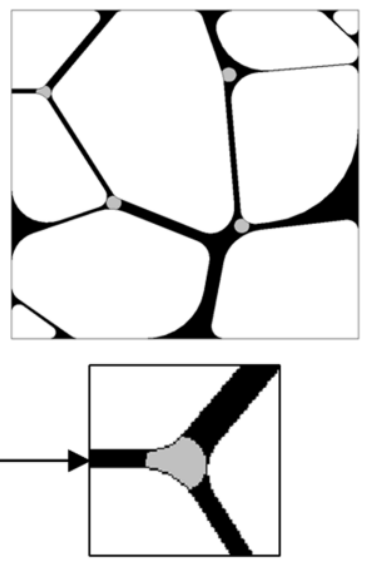

(d) 
Fig. 2: Variation in flow stress with temperature for the experimental data from [18], and the base-line [31] and enhanced Ludwik equations; $\dot{\varepsilon}=0.0015 \mathrm{~s}^{-1}$.

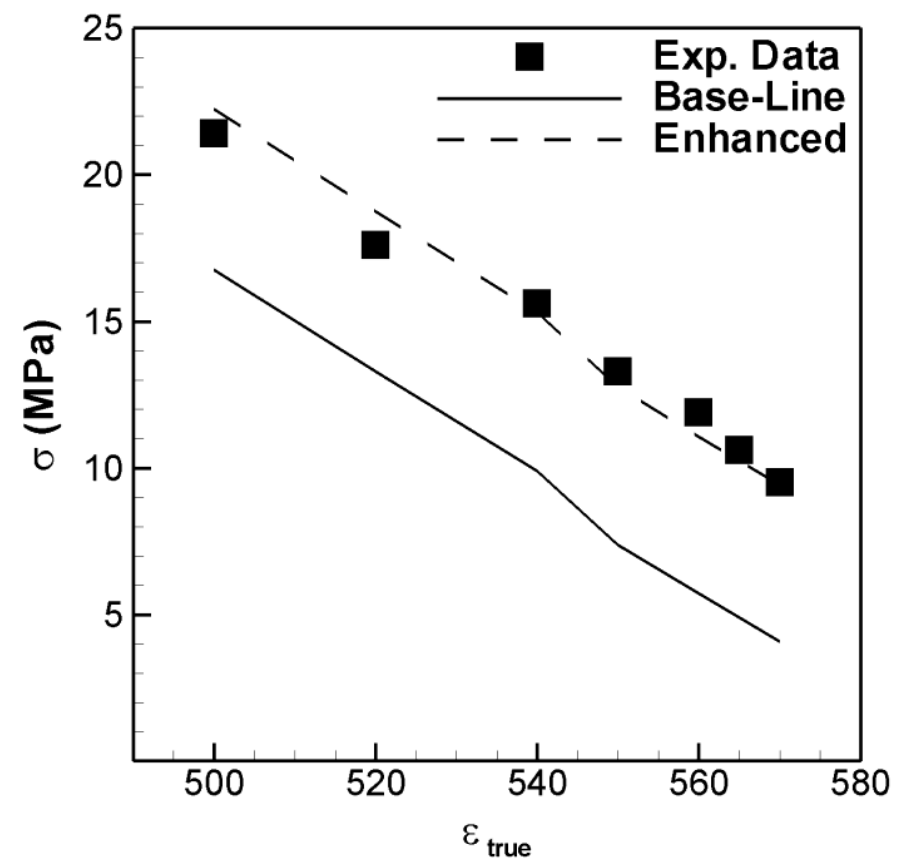

Fig. 3: Effect of fraction solid on the predicted semi-solid constitutive behavior in the range $0.75<f_{\mathrm{s}}<0.95\left(\bar{d}=225 \mu \mathrm{m}, f_{\mathrm{p}}=0\right)$.

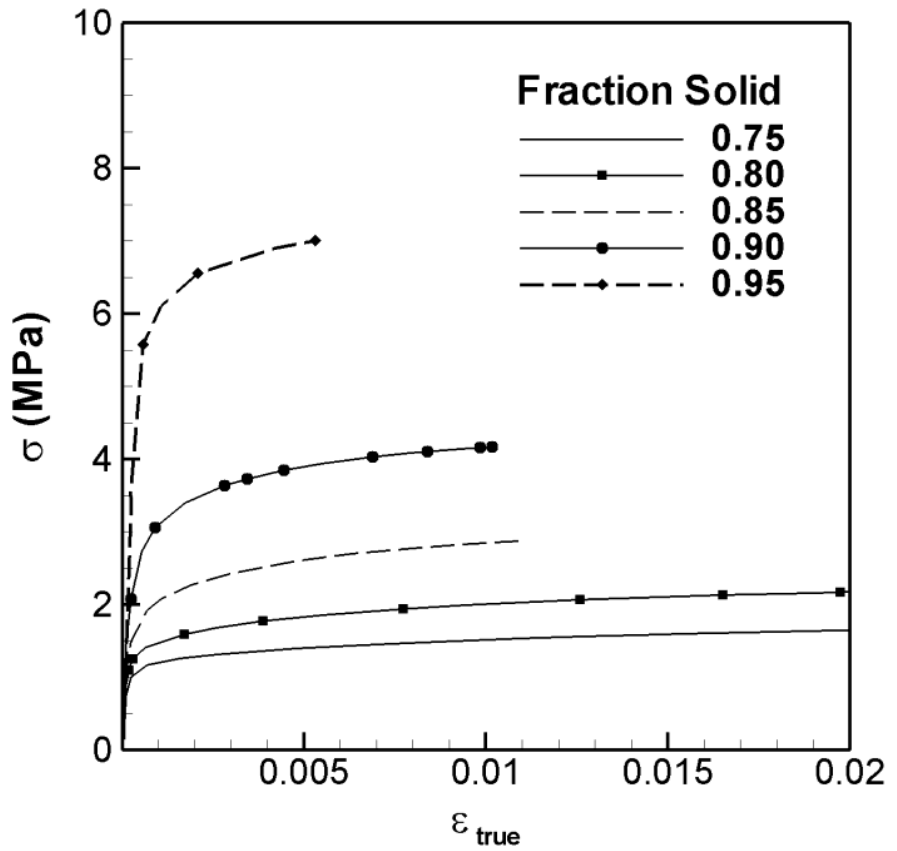


Fig. 4: Variation in predicted tensile response of the semi-solid material as a function of geometric configuration; $f_{\mathrm{s}}=\mathbf{0 . 9 0}, \bar{d}=150 \mu \mathrm{m}$, and $N_{\mathrm{g}}=\mathbf{5 6}$.

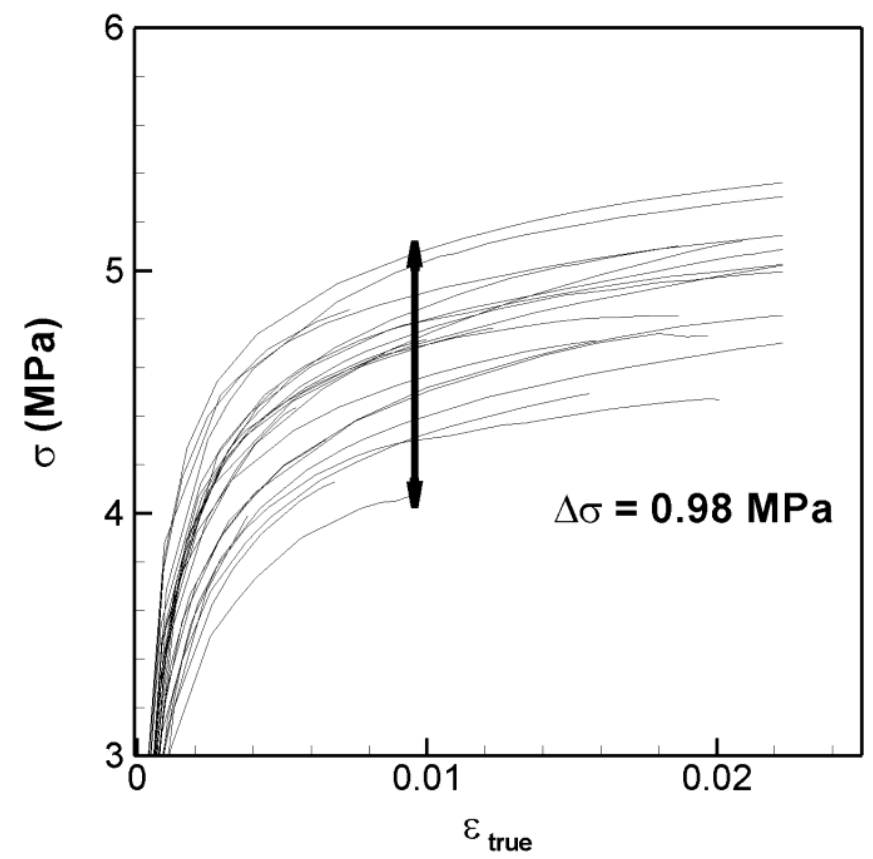

Fig. 5: Comparison of the predicted and experimentally measured semi-solid constitutive behavior of AA5182; $T=570^{\circ} \mathrm{C}, f_{\mathrm{p}}=0$, and $\bar{d}=225 \mu \mathrm{m}$. The inset graph on the right provides an enlargement of this comparison at small strains.

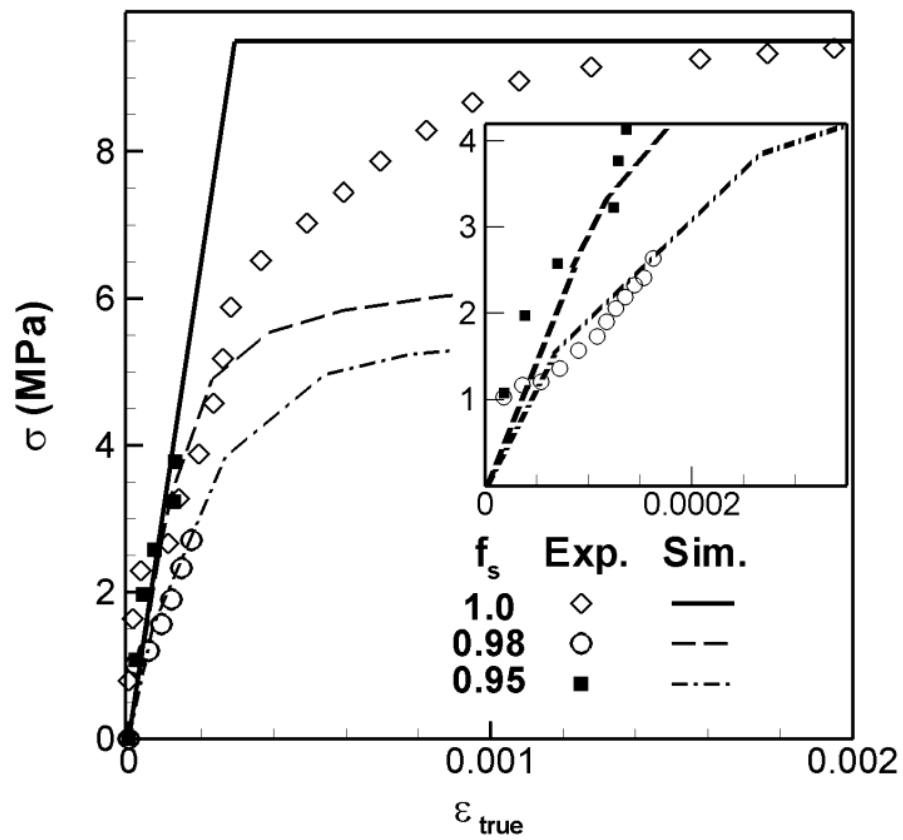


Fig. 6: Local strain vs. distance: (a) on the surface of a solidifying AA6111 alloy (after [24]), and (b) as calculated from the microstructure model; $f_{\mathrm{s}}=0.90, f_{\mathrm{p}}=0$, and $\bar{d}=150 \mu \mathrm{m}$.
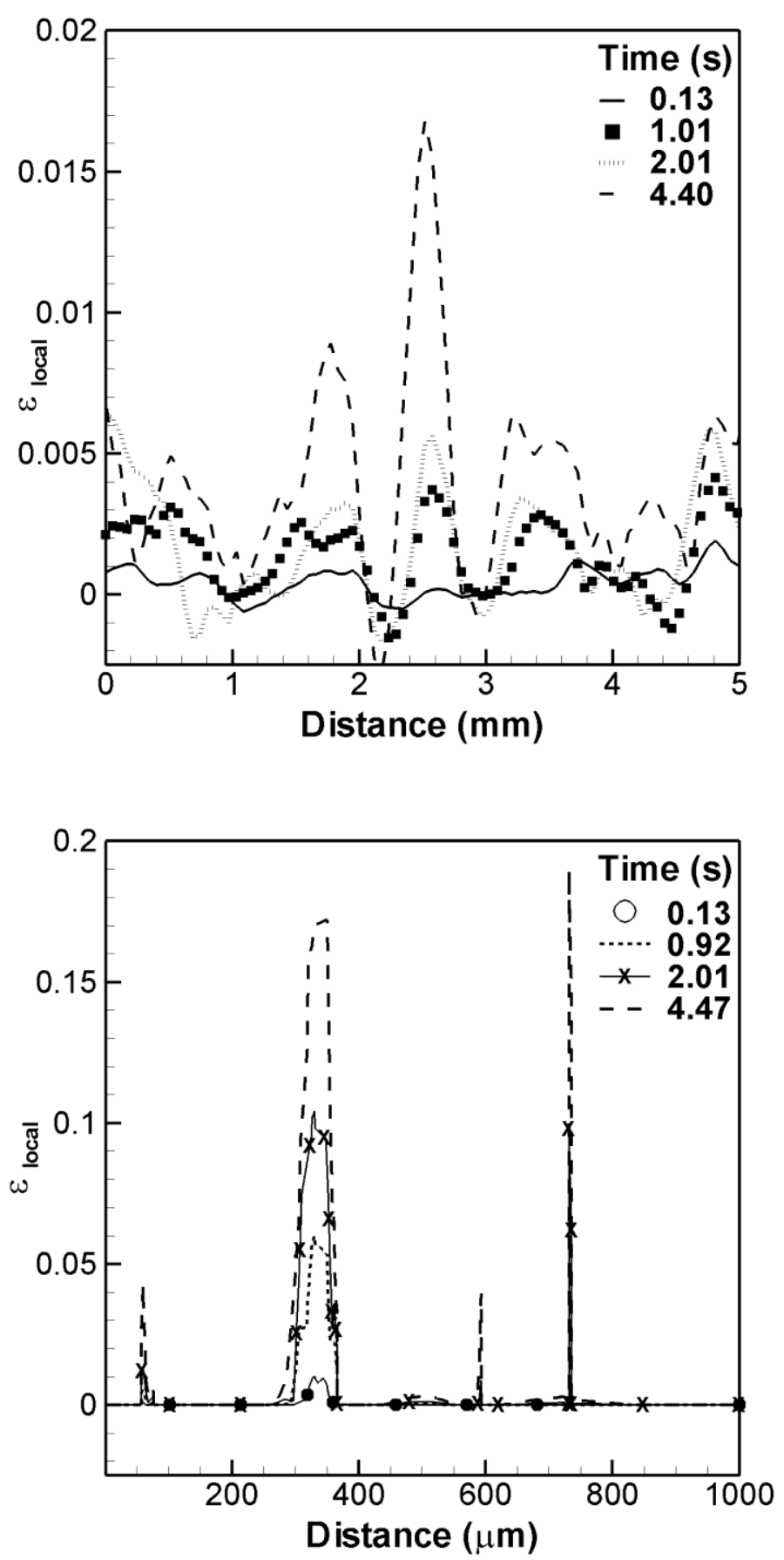
Fig. 7: Effect of: (a) grain size and (b) void area fraction on the predicted semi-solid constitutive behavior.
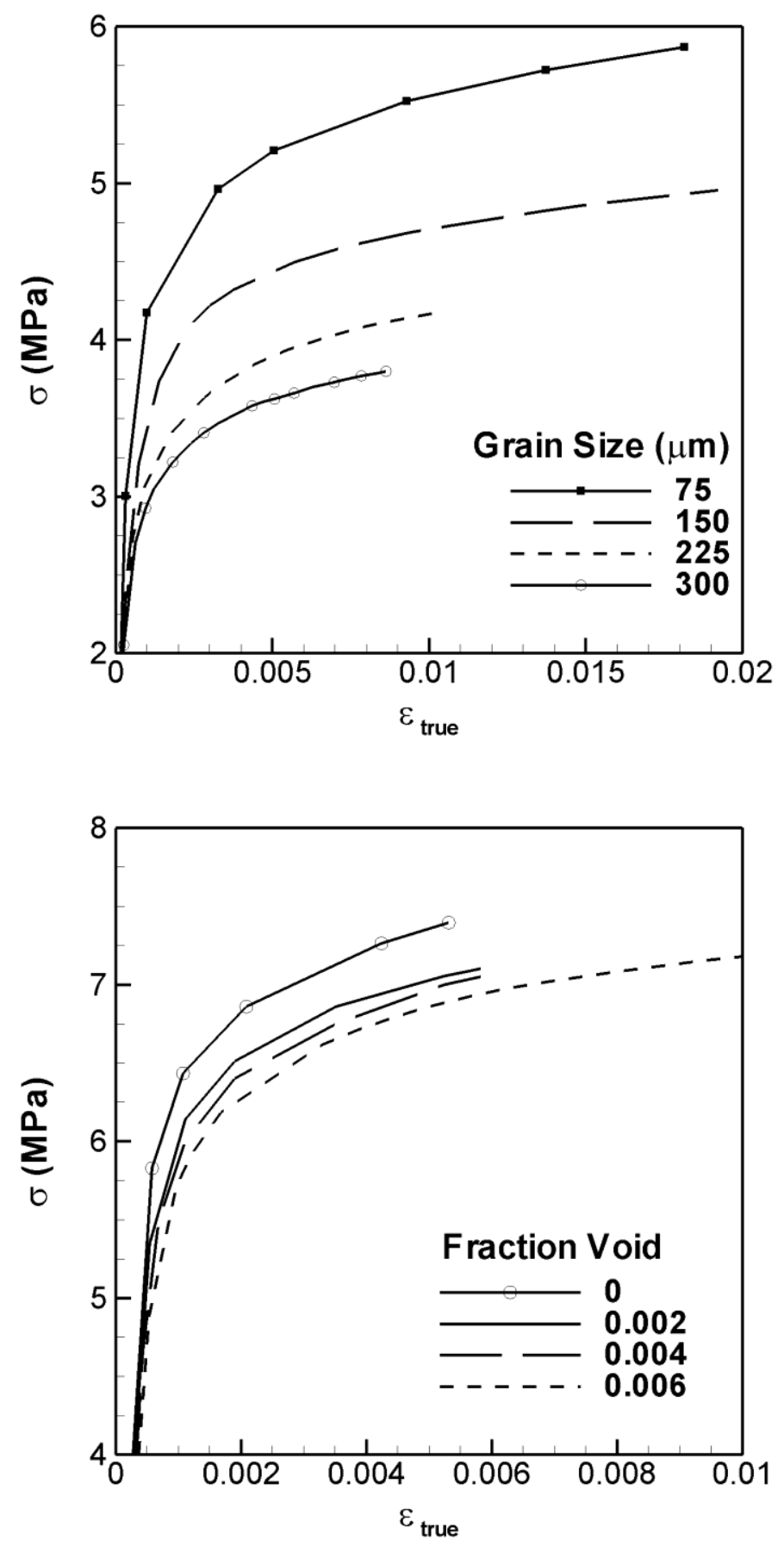
Fig. 8: Finite element simulation showing strain localization between voids $A$ and $B ; f_{\mathrm{s}}=$ $0.95, f_{\mathrm{p}}=0.004$, and $\bar{d}=150 \mu \mathrm{m}$.

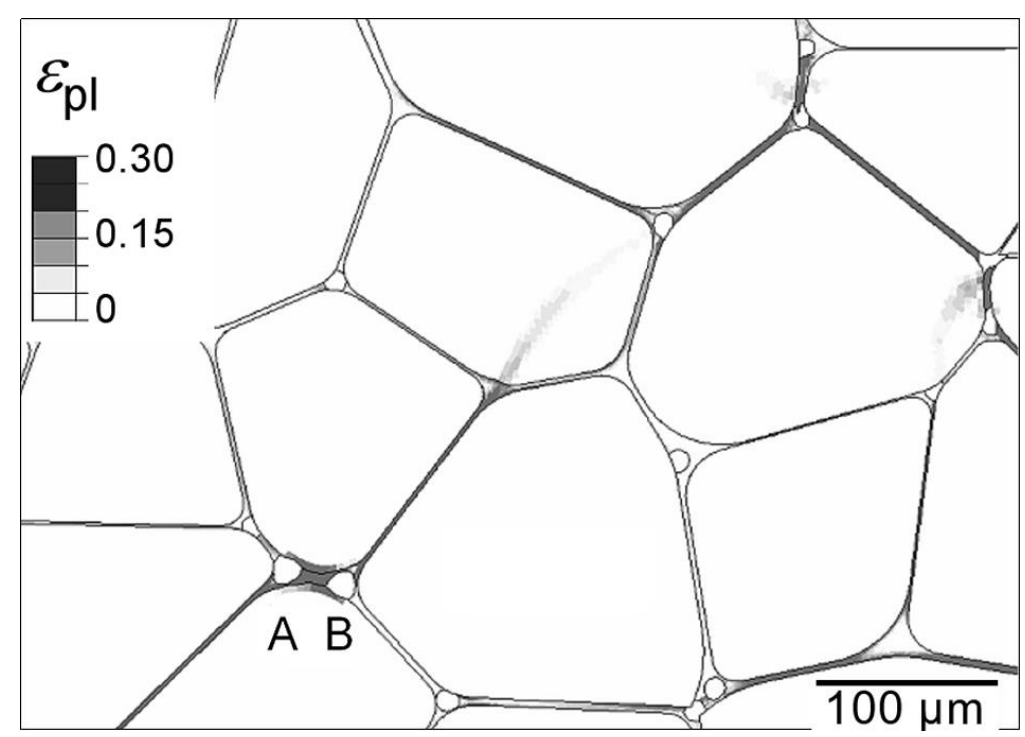




\section{References}

1. Gall, K., Horstemeyer, M., McDowell, D. L. and Fan, J., Mech of Mater 1999;32:277.

2. Pardoen, T. and Hutchinson, J. W., Acta Met 2002;51:133.

3. Lee, S. B., Rickman, J. M. and Rollett, A. D., Acta Mat 2006;55:615.

4. Lee, K. H., Moorthy, S. and Ghosh, S., Comput Method Appl Mech Eng 1999;172:175.

5. Gao, Y. X., Yi, J. Z., Lee, P. D. and Lindley, T. C., Acta Mat 2004;52:5435.

6. Brahme, A., Alvi, M. H., Saylor, D., Fridy, J. and Rollett, A. D., Scripta Mat 2006;55:75.

7. Rollett, A. D., Lee, S. B., Campman, R. and Rohrer, G. S., Annu Rev Mater Res 2007;37:627.

8. Rollett, A. D., Campman, R. and Saylor, D., Mater Sci Forum 2006;519-521:1.

9. Mathier, V., Jacot, A. and Rappaz, M., Modelling Simul Mater Sci Eng 2004;12:479.

10. Vernede, S., Jarry, P. and Rappaz, M., Acta Mat 2006;54:4023.

11. Vernede, S. and Rappaz, M., Acta Mat 2007;55:1703.

12. Lahaie, D. J. and Bouchard, M., Metall Mater Trans B 2001;32:697.

13. Larouche, D., Langlais, J., Wu, W. and Bouchard, M., Metall Mater Trans B 2006;37B:431.

14. Dijkstra, W. O., Vuik, C., Dammers, A. J. and Katgerman, L., In: Solid Proc \& Microst:

Symp Hon of W Kurz, M. Rappaz, C. Beckermann, R. Trivedi, Eds., Charlotte, NC (TMS, 2004), 151.

15. Eskin, D. G., Suyitno and Katgerman, L., Progress in Materi Sci 2004;49:629.

16. Fabregue, D., Deschamps, A., Suery, M. and Drezet, J. M., Acta Mat 2006;54:5209.

17. Ludwig, O., Drezet, J. M., Martin, C. L. and Suery, M., Metall Mater Trans A 2005;36A:1525.

18. Phillion, A. B., Cockcroft, S. L. and Lee, P. D., Mat Sci and Eng A 2008:doi:10.1016/j.msea.2008.01.078.

19. Colley, L. J., Wells, M. A. and Maijer, D. M., Mater Sci Eng A 2004;386:140.

20. van Haaften, W. M., Kool, W. H. and Katgerman, L., Mater Sci Eng A 2002;336:1.

21. Dahle, A. K. and Arnberg, L., Acta Met 1997;45:547.

22. Instone, S., St.John, D. H. and Grandfield, J., Int J Cast Met Res 2000;12:441.

23. Phillion, A. B., Cockcroft, S. L. and Lee, P. D., Scripta Mat 2006;55:489.

24. Mitchell, J. B., Cockcroft, S. L., Viano, D., Davidson, C. J. and St John, D., Metall Mater

Trans A 2007;38:2503.

25. Rappaz, M., Drezet, J. M. and Gremaud, M., Metall Mater Trans A 1999;30:449.

26. Suyitno, Kool, W. H. and Katgerman, L., Mater Sci Forum 2002;396-402:179.

27. Barber, C. B., Dobkin, D. P. and Huhdanpaa, H. T., ACM Trans Math Soft 1996;22:469.

28. Thompson, S., Cockcroft, S. L. and Wells, M. A., Mater Sci Techn 2004;20:497.

29. Phillion, A. B., Lee, P. D., Maire, E. and Cockcroft, S. L., Metall Mater Trans A 2008:[Under Review].

30. Phillion, A. B., Hot Tearing and Constitutive Behaviour of Semi-Solid Aluminum Alloys, PhD, University of British Columbia (2007). 
31. Chaudhary, A. and Wells, M. A., In: Proc Intl Conf Adv in Mater \& Meter Process, U. K. Chatterjee, B. K. Dhindaw, Eds., IIT Kharagpur, India (2006), 763.

32. Ludwig, O., Drezet, J. M., Meneses, P., Martin, C. L. and Suery, M., Mater Sci Eng A 2005;413:174.

33. Venkatramani, G., Ghosh, S. and Mills, M., Acta Mat 2007;55:3971.

34. Henning, M. and Vehoff, H., Mater Sci Eng A 2007;452:602.

35. Bower, A. F. and Wininger, E., J Mech Phys Solids 2004;52:1289.

36. Huber, G., Brechet, Y. and Pardoen, T., Acta Mat 2005;53:2739. 